\title{
Highlights from the IceCube collaboration: From astrophysics to dark matter
}

\author{
Juan A. Aguilar* for the IceCube Collaboration \\ Université Libre de Bruxelles \\ E-mail: aguilareicecube.wisc.edu
}

\begin{abstract}
Since its completion in 2010, the IceCube Neutrino Observatory has produced several important scientific results that extend from astroparticle physics and multi-messenger astronomy to the indirect search for dark matter as a particle. Two of the major milestones of the IceCube collaboration are the detection in 2013 of a flux of high-energy neutrinos incompatible with the expected background fluxes, and the observation of a high energy neutrino in coincidence with a flaring blazar. These achievements have heralded the birth of neutrino astronomy and consolidated multi-messenger astronomy as new field within the context of observational astroparticle physics. In addition to its astrophysical contributions, IceCube has also a lively program on dark matter searches with very competitive results for spin-dependent cross-sections. In these proceedings I will review the recent highlights from IceCube which includes the recent observation of TXS-0506-056 as a possible neutrino source.
\end{abstract}

2nd World Summit: Exploring the Dark Side of the Universe

25-29 June, 2018

University of Antilles, Pointe-à-Pitre, Guadeloupe, France

\footnotetext{
*Speaker.
} 


\section{Introduction}

The IceCube Neutrino Observatory consists of an array of 5,160 photomultipliers tubes (PMTs) installed among 86 strings and deployed at $1.5-2.5 \mathrm{~km}$ deep within the Antarctic ice cap at the South Pole. This large instrumented volume of $1 \mathrm{~km}^{3}$ measures the Cherenkov light induced by relativistic charge particles produced by neutrino interactions with the matter surrounding the detector. The main scientific goal of IceCube is the detection of astrophysical neutrinos emitted by the same sources responsible for the production of cosmic rays. Neutrinos are one special messenger for the observation of the high-energy Universe and they play a significant role in multi-messenger astronomy. Contrary to cosmic rays, whose random-walk through the intergalactic and galactic magnetic fields spoils all directional information, neutrinos are electrically neutral and are not deviated by magnetic fields. Also, unlike gamma rays which cascade down in the extragalactic background light and the Cosmic Microwave Background (CMB), neutrinos are weakly interacting particles and can reach the Earth from anywhere in the Universe without interacting. In these proceedings I will review the latest results from the IceCube collaboration both in the search of astrophysical neutrinos and the search for dark matter.

\section{Astrophysical Neutrinos}

The search for astrophysical neutrinos is broadly divided into two categories. In the diffuse search it is assumed that the sources of neutrinos are faint but numerous so that the overall diffuse neutrino emission from all sources can be observed. Fermi shock acceleration models predict a neutrino spectrum harder than those of atmospheric neutrinos and muons, therefore diffuse searches try to identify an astrophysical emission at higher energies, above the steeply falling spectrum of the atmospheric background. The second category is the so-called point source searches. These searches test the hypothesis where a few bright sources are capable of emitting enough neutrinos to produce an accumulation of events observable at a certain location within IceCube's visible sky. For these searches, a good angular resolution is key in order to reject the uniformly distributed background of atmospheric muons and neutrinos. Also within the context of point sources searches, external information from other observatories can be used as a way to limit the search region temporally or spatially and therefore limit the background and the effective number of trials.

\subsection{Diffuse emission: the astrophysical neutrino flux}

In 2013 IceCube detected for the first time a component of astrophysical neutrinos. A selection of high-energy starting events inside the fiducial volume of IceCube called HESE (High-Energy Starting Events) [1] was used for this analysis. This discovery was later confirmed with a selection of through-going events where only up-going tracks coming through the Earth are selected as a way to reject the large contribution of down-going atmospheric muon background events. In both cases, the astrophysical component appeared clearly as an excess of events at the highest energies above the spectrum of atmospheric neutrinos. To date 103 of these HESE events have been recorded in 7.5 years with 60 of them depositing more than $60 \mathrm{TeV}$ of energy in the detector.

The spectrum of these events was fitted with a likelihood method in the energy range of $60<$ $E_{v}<10 \mathrm{PeV}$ using an unbroken-power law with a variable spectral index. The result of the fit are 
shown in Fig. 1 together with the best fit obtained with the through-going muon sample [2]. As can be seen both results are comparable at $>200 \mathrm{TeV}$, while some tension exists between the best fit slopes for the two different datasets suggesting a second astrophysical component or a broken power-law spectrum. However, given the limited statistics and its energy range, the HESE data sample cannot distinguish between different astrophysical flux models at this moment [1].

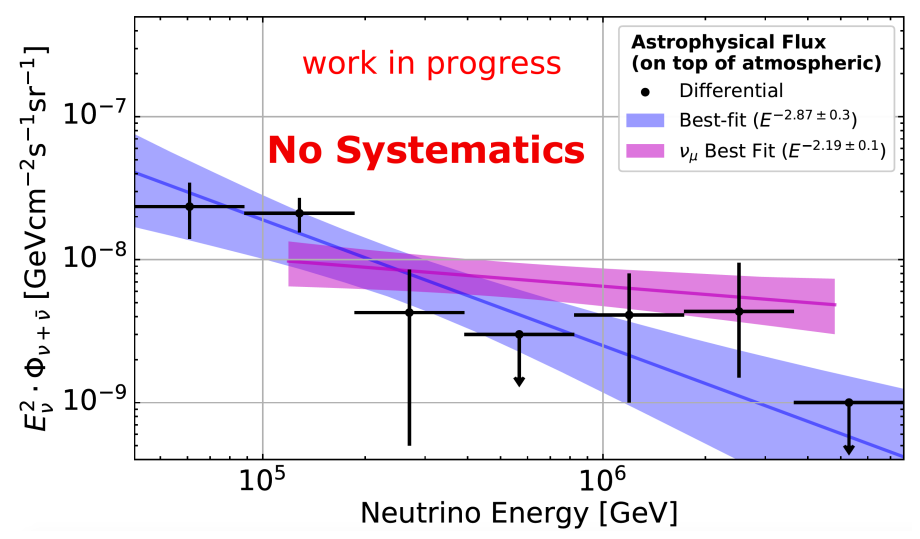

Figure 1: Astrophysical neutrino flux as a function of energy. The black points with error bars are extracted from a combined likelihood fit of all background components and a cosmic neutrino flux with independent normalization in each energy band (assuming an $\mathrm{E}^{-2}$ spectrum within the band). Error bars indicate the $\Delta \mathscr{L} \pm 1$ contours of the flux in each energy bin. The single power law fit result is shown in blue, the pink band shows the 8yr high-energy up-going muon best fit.

In typical acceleration models of cosmic-rays neutrinos are produced as decay products of pions and muons yielding a flavor composition of $v_{e}: v_{\mu}: v_{\tau} \simeq=1: 2: 0$. For an astrophysical neutrino flux over long baseline distances, neutrino oscillations will degenerate this composition to an equal mix $v_{e}: v_{\mu}: v_{\tau}=1: 1: 1$. The different event signatures in the IceCube detector can provide information about the flavor composition of the astrophysical neutrinos. For example, only muons produced via charge current (CC) $v_{\mu}$ interactions are long-ranged enough to leave behind a track-like signature in the detector. On the other hand, $\mathrm{CC}$ interaction of electron neutrinos and neutral current (NC) of all other flavors will produce electromagnetic and hadronic showers that result in a cascade-like topology. Tau neutrinos can also produce a very unique signature where for deposited energies of $E_{d e p}>100 \mathrm{TeV}$ the resulting $\tau$ from the neutrino interaction CC can travel enough distance in the detector to leave a visible track before decaying. About $83 \%$ of the $\tau$ decays will result in a new $v_{\tau}$ appearance with a second cascade in what is called a double-cascade topology. A recent analyses has resulted in a best fitted flavor composition of $v_{e}: v_{\mu}: v_{\tau}=1.05$ : $1.35: 0.6$ where two candidates events of double cascade signature were identified.

\subsection{Point sources searches}

Despite the study and the characterization of the diffuse astrophysical flux, the origin of these neutrinos is not yet known. Clustering analyses performed on the HESE sample are thus far unable to resolve statistically significant hot spots, or areas of events accumulation beyond the expectation of an isotropic flux. As already mentioned, point source searches can benefit by the inclusion of external information that can be used to limit the number of trials factors by, for example, looking 
at the direction of a strong gamma-ray counterpart. This multi-messenger strategy is integrated in a real-time framework [3] in which information about different observations is shared in a near real-time among different observatories.

\subsection{Real-time alerts: the TXS $0506+056$}

Operating since 2016 IceCube has established the real-time alert system that rapidly notifies the astronomical community of the direction of astrophysical neutrino event candidates [3]. These type of alerts include HESE track-like events as well as very high-energy through-going events with energies in the $500 \mathrm{TeV}$ to $10 \mathrm{PeV}$ range (the Extremely High Energy neutrino alert). Since the beginning of the program, 11 events alerts have been issued. One of these alerts corresponded to the IceCube event IceCube-170922A on September 22, 2017 with an estimated energy of $\sim 290$ $\mathrm{TeV}$. Figure ?? shows a representation of this event where the best fitted direction is also indicated. Four days later, the Fermi Large Area Telescope (LAT) Collaboration reported that the direction of origin of IceCube-170922A was consistent with the known blazar TXS 0506+056 which was at that time in a flaring state (Fig. 2).
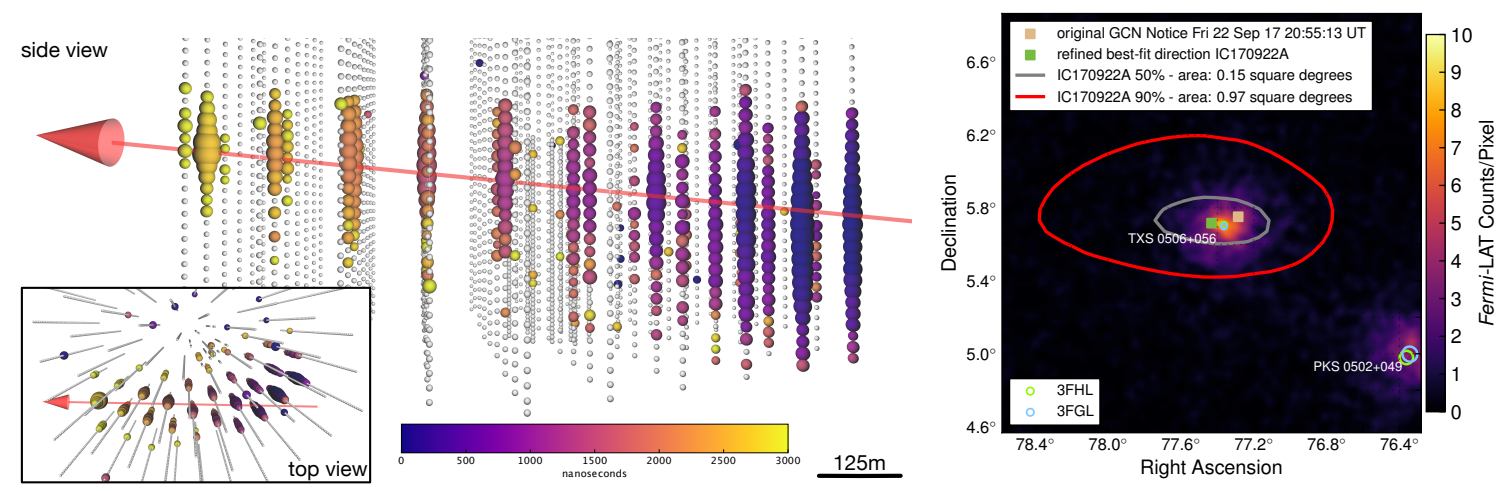

Figure 2: Left: Event display for neutrino event IceCube-170922A. The time at which each photomultiplier observed a signal is reflected in the color of the hit, with dark blues for earliest hits and yellow for latest. The best-fitting track direction is shown as a red arrow. Right: Fermi-LAT observation of the IceCube-170922A's region.

Prompted by the Fermi-LAT observation the Major Atmospheric Gamma Imaging Cherenkov (MAGIC) observed the location starting on 28 September 2017 after a previous, and null, observation on 24 September under not optimal observation conditions. This second time, MAGIC detected an excess of photons from TXS 0506+056 at energies up to about $400 \mathrm{GeV}$. Given these observations an extensive multi-wavelength campaign followed. The chance coincidence of an IceCube neutrino flare, and finding a blazar in a flaring state at the same location has been disfavored at the level of $3 \sigma$. This coincidence thus suggests that $\gamma$-ray blazars are strong candidates for the origin of at least a fraction of the observed astrophysical neutrinos in IceCube [4].

\section{Dark Matter searches}

The IceCube neutrino telescope can also provide interesting limits regarding the existence of Dark Matter (DM) as a particle. Indirect detection of DM uses the annihilation or decay products 
of DM particles as traces to prove its existence. Search strategies usually focus on finding an excess of neutrinos at locations in the sky where reservoirs of DM are believed to accumulate. These overdensities of DM are typically formed in two processes: they can be part of the DM relic density that seeded the structure formation of the galaxy (such as the Galaxy Center) or are created by a capturing process by local massive celestial bodies like the Sun or the Earth.

\subsection{Dark Matter annihilation from the Sun and Earth}

If DM halo particles interact with Standard Model particles they can undergo scattering with nucleons of the Sun or Earth loosing enough energy to be gravitationally captured. This process implies that, over time, an excess of DM will accumulate at the center of these objects. Under these high density conditions, DM will annihilate producing as final products stable and light particles like neutrinos and $\gamma$-rays. Given the high density of the Sun and Earth, only neutrinos will escape, and hence DM searches from the center of these objects is only accessible via neutrino telescopes. There is however a significant difference between the Sun and Earth capturing processes. The time required to reach an equilibrium (where capturing rate equals the annihilation rate) is, for values of $\sigma_{\chi p}^{S I} \sim 10^{-43} \mathrm{~cm}^{-2}$, of the order of $10^{11}$ years, which is much longer than the age of the Earth. For the Sun, however, the time for equilibrium is smaller than the age of the solar system, and therefore it can be assumed that DM accumulation and annihilation in the Sun has reached an equilibrium. The equilibrium condition makes it possible to translate an upper limit on the DM induced neutrinos from the Sun to a limit on $\sigma_{\chi p}$ (Fig. 3), while for the Earth an annihilation cross-section needs to be assumed.

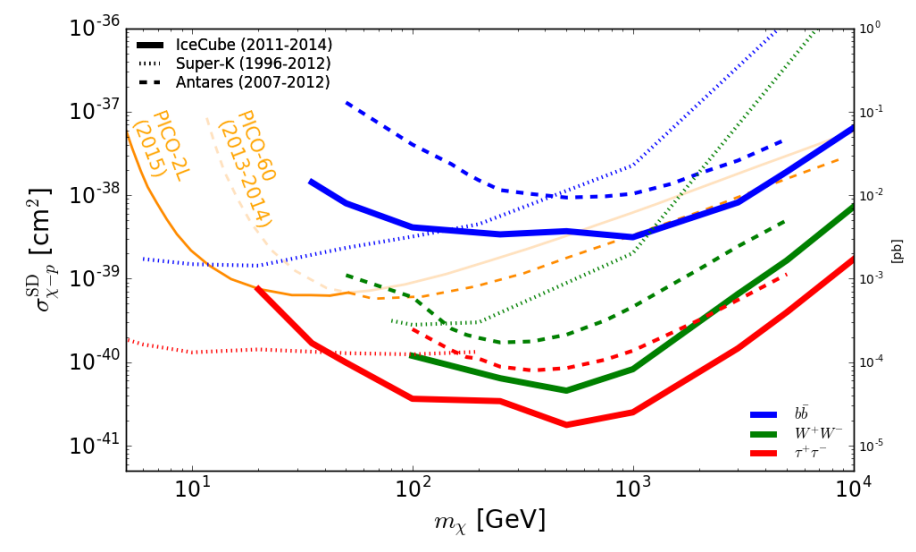

Figure 3: Limits on the spin-dependent WIMP-proton cross-section $\sigma_{\chi-p}^{S D}$ from the Sun compared to results from other neutrino detectors and direct detection experiments [5].

\subsection{Dark Matter from the center of the Galaxy}

With the formation of structures in the Universe, over-fluctuations of the ambient relic DM density also developed. The study of kinematics of stars in galaxies together with N-body simulation predict an excess of DM at the center of galaxies. The inner Galactic region is therefore one of the most interesting regions to search for DM since most galactic halo density profiles models show an excess of DM towards its center. Unfortunately, the Galactic Center is also one of the regions with multiple $\gamma$-ray sources, and therefore the astrophysical background is considerable 
when doing searches with $\gamma$-rays. For neutrinos, the Galactic Center is also accessible with a much lower background although significant lower signal predictions as well. Figure 4 shows the upper limits on the velocity averaged DM annihilation cross-section from different analyses in IceCube considering different event selections.

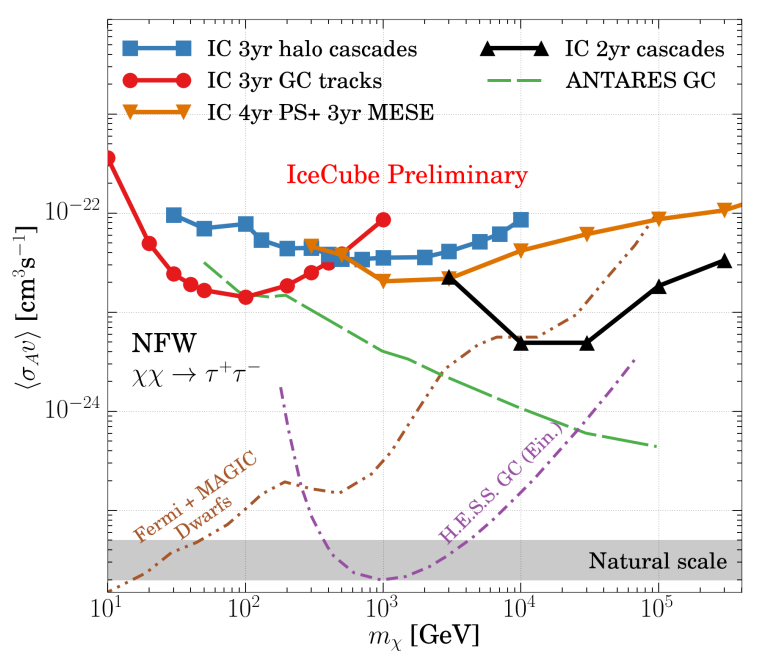

Figure 4: Upper limits on the $\left\langle\sigma_{A} \mathrm{v}\right\rangle$ as function of the WIMP mass for the annihilation channel $\chi \chi \rightarrow \tau \tau$ assuming the Navarro-Frenk-White halo profile [6].

\section{Conclusions}

The IceCube Collaboration is currently characterizing the properties of the observed astrophysical neutrino flux such as the spectrum and flavor composition, while at the same time looking for the sources of these neutrinos. The real-time framework deployed in 2016 has given an significant indication of a correlation of a high-energy neutrino event in spatial and temporal coincidence with a flaring blazar. At the same time, IceCube continues its quest to search for dark matter. These searches can access regions which are blind for gamma-ray astronomy such as the Sun and the Earth and are complementary to direct detection strategies.

\section{References}

[1] IceCube collaboration, Science, 342 (6161) 2013

[2] IceCube, PoS (ICRC 2017) 1005

[3] IceCube collaboration, Astropart. Phys. 92, 30 (2017)

[4] The IceCube Collaboration, Fermi-LAT, MAGIC, et al., Science 361, eaat1378 (2018)

[5] IceCube collaboration, Eur. Phys. J. C (2017) 77: 146

[6] IceCube collaboration, Eur. Phys. J. C (2017) 77: 627 